\title{
Virtual Models Obtained via Intraoral Scanning as Alternated to Clinical Evaluation and $\beta$-Hemihydrate Plaster Models
}

\author{
CORINA MARILENA CRISTACHE ${ }^{1,2}$, EUGENIA EFTIMIE TOTU3*, IRINA ADRIANA BEURAN ${ }^{4}$, ELENA MIHAELA CARAUSU5, \\ TIBERIU TOTU6, LILIANA BURLIBASA \\ ${ }^{1}$ Carol Davila University of Medicine and Pharmacy, Faculty of Midwifery and Medical Assisting (FMAM), Department of Dental \\ Techniques, 8, Eroilor Sanitari Bvd., 050474 Bucharest, Romania \\ ${ }^{2}$ Concordia Dent Clinic, 7D-7E Vitan-Barzesti St., Bucharest, Romania \\ 3University Politehnica of Bucharest, Faculty of Applied Chemistry and Material Science, 1-5 Polizu Str., 11061, Bucharest, \\ Romania \\ ${ }^{4}$ Carol Davila University of Medicine and Pharmacy, Faculty of Dental Medicine,19 Plevnei Str., Bucharest, Romania \\ ${ }^{5}$ Grigore T Popa University of Medicine and Pharmacy, Faculty of Dental Medicine, 16 University Str., 700115, Iasi, Romania \\ ${ }^{6}$ C.M.M.I.P., University of Politehnica of Bucharest, 313 Splaiul Independentei , 77206, Bucharest, Romania \\ 7University of Bucharest, Faculty of Biology, 91-95 Splaiul Independentei, 050095, Bucharest, Romania
}

\begin{abstract}
Digital scanners are currently use in various fields of dentistry such as prosthodontics, dental implantology, orthodontics, with a high degree of accuracy, mostly for replacing conventional impressions. The aim of the present study was to assess the accuracy (trueness and precision) of gingival margin measurements, on virtual models obtained by intraoral scanning, for precise diagnosis and treatment monitoring of periodontal disease. Three methods were compared for accuracy: clinical measurements using periodontal probes, digital intraoral scanning and measurements in MeshLab software and 2D digital measurements on intraoral photography taken during clinical measurements. Intraoral scanning was proved to be a reliable method for measuring the gingival level for diagnosis and clinical monitoring having a good accuracy as compared to the gold standard (clinical measurements).
\end{abstract}

Keywords: gingival margin intraoral scanning, periodontal disease, treatment outcome

Intraoral scanning or direct optical survey of the oral structures, especially teeth and surrounding soft tissue, became largely used lately due to the increased accuracy and the great progresses registered by the digital technology in the last years.

Clinical oral examination, with recognition and pathology description for documentation, communication between clinicians and follow-up for evaluation of lesion changes, is considered the gold standard. However, clinical assessment is subjective and dependent on clinician's experience [1].

In order to document difficult cases, to keep the record on treatment effectiveness and also to measure recession without interference of soft tissue, plaster models are useful diagnostic tools. When periodontal disease is present and teeth are mobile, a correct impression could lead to accidentally teeth removal. Also, despite of the improvements in dental materials, dental gypsum, used for study models manufacturing, had a significant number of drawbacks such as physical and chemical damage, wear and tear, distortion and is not cost-effective [2]. Thus, in late 1990'digital models, obtained via intraoral scanning or scanning plaster models, were introduced, with the great advantage of electronic transfer and storage.

Digital scanners are currently used in various fields of dentistry such as prosthodontics, dental implantology, orthodontics, with a high degree of accuracy, mostly for replacing conventional impressions. Moreover, the distortion, risk of infection spreading and patient discomfort caused by the conventional impressions, are limited. In orthodontics, digital scanners and virtual models are used to evaluate the outcome of the treatment.

In the last years, intraoral scanning was more and more used to assess the length and volume of gingival recessions
[2-5], or to document soft tissue shape and irregularity [1].

For assessing periodontal disease occurrence and evolution, also for monitoring the efficacy of the treatment, digital scanning could replace conventional stone casts and intraoral two-dimensional photographs.

The aim of the present study was to assess the accuracy (trueness and precision) of gingival margin measurements, on virtual models obtained by intraoral scanning, for precise diagnosis and treatmentmonitoring of periodontal disease.

\section{Experimental part}

Nowadays, for an accurate documenting of clinical cases in dental medicine, the plaster models are widely used. A replica/a model of the oral cavity could be obtained using various dental materials. For an integral model, usually a so-called refractory material is applied, which is able to maintain the shape and strength, being chemically and physically stable over a large range of temperatures. The well-known gypsum represents the base of such materials.

Gypsum is calcium sulphate dihydrate $\left(\mathrm{CaSO}_{-}-2 \mathrm{H}_{2} \mathrm{O}\right)$ and it is crystalline with a spatial structure as shown in Figure 1.
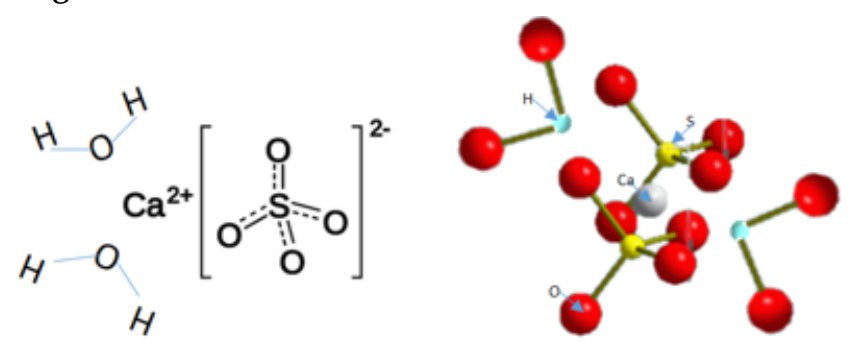

Fig. 1. Gypsum model.

\footnotetext{
* email: eugenia_totu@yahoo.com All the authors have equally contributed to the manuscript, and they should be regarded as main authors.
} 
The dehydration process of gypsum involves three steps, namely: a) the break of the hydrate bonds with water molecules participating; $b$ ) the diffusion of the water molecules through the crystals; c) the transport of the water molecules through pore spaces. When heated, gypsum loses its hydration water in the presence of open air.

When mixed with water, the gypsum could be milled resuming its stone-like state. As consequence, it could be shaped and hardened according to the desired characteristics. It is interesting that gypsum is able to follow a closed loop(Fig. 2) which allows its recycling.

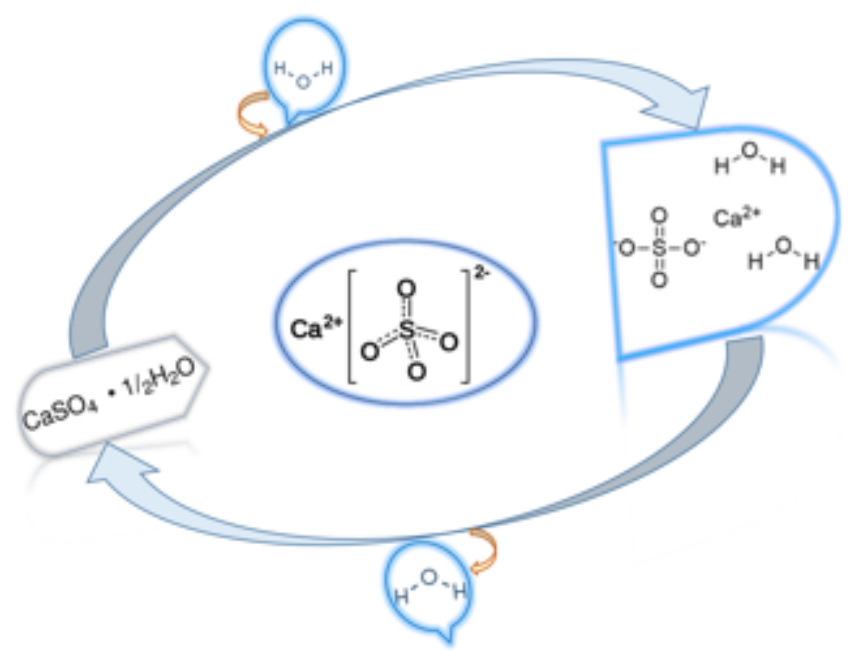

Fig. 2. Recycling loop for gypsum

Depending on the immediate purpose, some additives could be introduced. Among these, the most importantare $\mathrm{K}_{2} \mathrm{SO}_{4}$ (accelerator), $\mathrm{NaCl}$ (generating supplementarysites for nuclei growth), or $\mathrm{Na}_{2} \mathrm{~B}_{4} \mathrm{O}_{7} .10 \mathrm{H}_{2} \mathrm{O}$ (crystallization delay/ preventing).

The morphology of the resulting $\beta$-hemihydrate was studied by help of scanning electronic microscopy (SEM) using an Oxford Instruments equipment.

However, there are major drawbacks of the gypsum, like volumetric changes or physical modifications. In such context, we propose digital models obtained via intraoral scanning as viable alternative for replacing the $\beta$ hemihydrate plaster based models.

Ten patients out of the fifty participants of the randomized control clinical trial registered with ClinicalTrials.gov Identifier:NCT03656484 and approved by the Romanian Research Bioethical Committee (No. 176/ 2018) have been selected for participating in this study. The following inclusions criteria were applied: patients diagnosed with mild periodontal disease, according to Eke and co-workers as two or more interproximal sites with clinical attachment loss (CAL) $\geq 3 \mathrm{~mm}$ and $\geq 2$ interproximal sites with probing depth (PD) $\geq 4 \mathrm{~mm}$ (not on the same tooth) or one site with PD $\geq 5 \mathrm{~mm}$ [6]; with at least one natural, not restored tooth in the maxillary/mandibular anterior region (central incisor to first premolar); age $\geq 30$ years old; no prior periodontal treatment and with good general health. Informed consent was obtained from all participants included in this study.

As conventional method, the direct clinical measurements of the gingival margins using a millimeter grade calibrated PCP15 probe (Henry-Schein Dental, Melville, NY, USA), was performed by two independent investigators, from the clinical gingival margin to the cemento-enamel junction (CEJ), at the buccal site, parallel to the long axis of the tooth. Three successive measurements, documented by intraoral photographs, for a precise evaluation, were made for each patient.

The digital 3D measurements were executed by the same two independent investigators, employing the free MeshLab software (Visual Computing Lab, Institute of Science and Technology Italy), on digital model obtained via intraoral scanning using the scanner Carestream 3600 (Carestream Dental LLC, Atlanta, GA, USA) (Fig. 3). All participants were scanned by the same investigator, experienced in using intraoral scanners, according to the scanning protocol provided by the manufacturer.

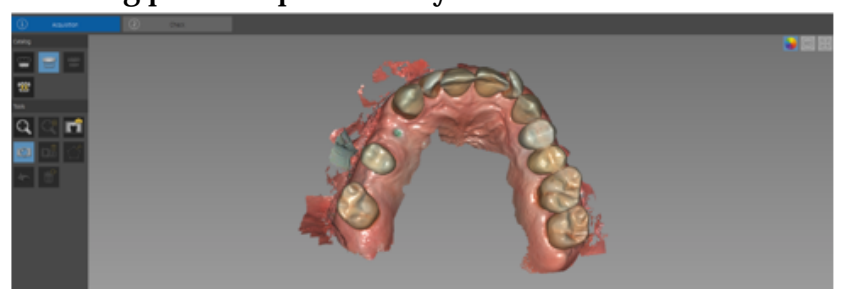

Fig. 3. Digital model obtained by intraoral scanning

All digital scans were exported as Polygon File Format (ply.) in MeshLab software (open access software) and three successive measurements for each selected tooth were done by each independent investigator, in a random order (Fig.4).

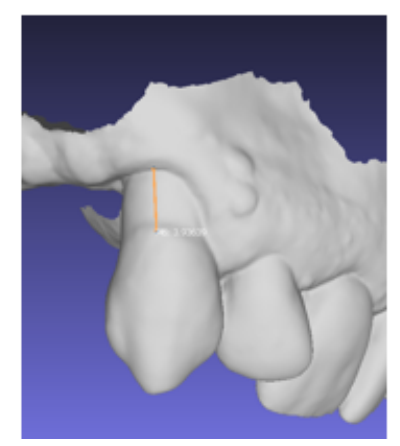

Fig. 4. Random measurements for selected tooth in 3D MeshLab software

The digital 2D measurements were performed as follows: the .jpeg format pictures obtained during clinical measurements were imported in Image J software (a) avabased image processing open program developed at the National Institutes of Health, USA) to measure, with a greater accuracy, the selected gingival margins. The conversions pixels to centimeters were performed with the aid of the millimeter gradations on the periodontal probe.

Patients were also questioned regarding their experience with intraoral scanning comparing to the clinical measurements and their answer were recorded using a 010 Visual Analogue Scale (VAS), were 0 means strongly preferred clinical assessment and 10 strongly preferred intraoral scanning.

All data were tabulated and statistical analyses were done in Excel (Microsoft Office 2019) and XLSTAT 2014 (Addinsoft, New York, NY, USA).

\section{Results and discussions}

Among the ten patients enrolled, aged between 47 and 59 years old (mean 58.10), five were female. 18 anterior teeth were considered. Two sets of 162 measurements: clinical, digital in 3D MeshLab software and digital in 2D on Image J software, were made.

Bland and Altman Plots were drawn to evaluate the correlation between the two examiners for each of the three different methods. The mean value of the three consecutive measurements performed for an individual tooth by every examiner was considered. In order to see if there is any difference between the two investigators for 


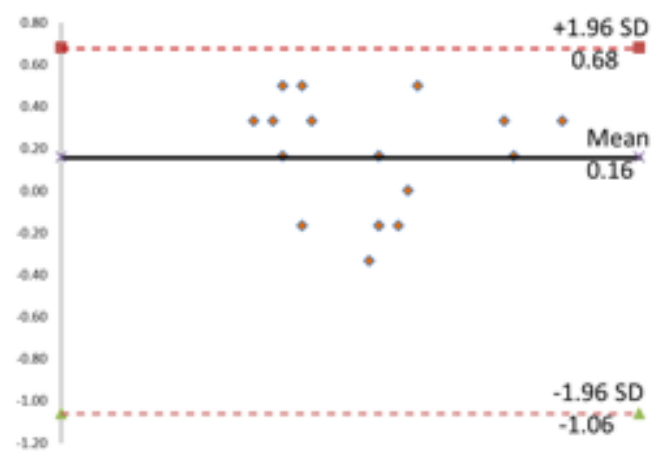

a

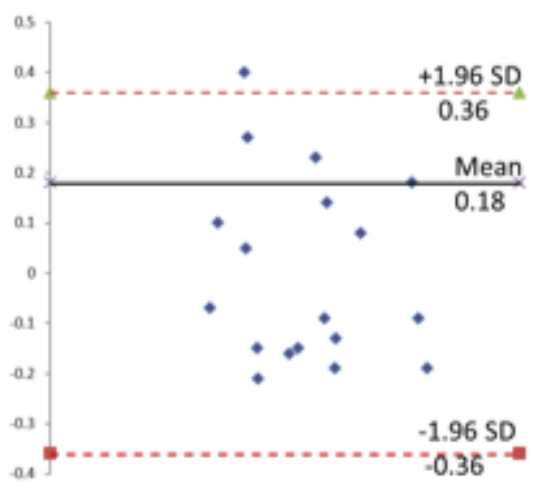

b

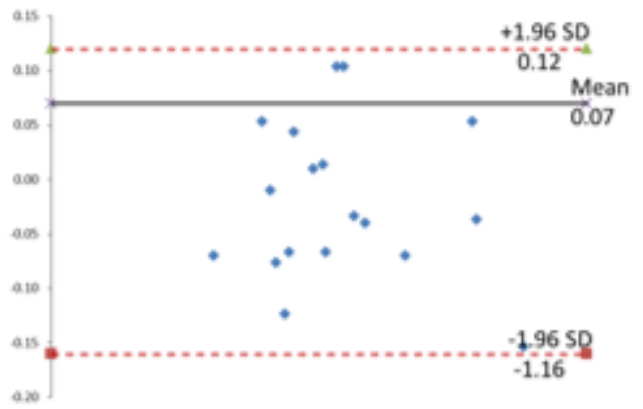

Fig. 5. Bland and Altman analysis showing the extent of agreement among the two examiners for (a) the clinical measurements, (b) 3D digital measurements on the models obtained by intraoral scanning and (c) digital 2D measurement on photographs with periodontal probe. The within-examiners and biases along with their $95 \%$ upper and lower limits for each method were also presented

each method (inter-examiner reliability), the means ot each pair of measurements ( $x$ value) were plotted against the difference between the measurements (y value). Bland and Altman Plots for agreement assessment with the use of clinical measurements, 3D measurements on intraoral scanning and digital 2D measurements are presented in figure 5 a-c.

As it could be observed in Figure 4, all the obtained values were cluster around the mean of the differences (the bias), and at least, within the two standard deviations of the mean (95\% prediction interval), meaning a strong agreement between the two investigators for each of the three individual methods [7].

The main goal of the present study was to investigate the reliability of digital measurements, therefore the pairwise variations between the three methods for the measurements of the gingival margins level was assessed.

The correlation between clinical and 3D digital measurement is illustrated in Fig. 6. As could be noticed from the scatter diagram, there is high correlation between the two methods and the correlation coefficient obtained using the Excel worksheet formula = CORREL (clinical, digital) is 0.97 .

\section{Correlation Clinical vs Digital}

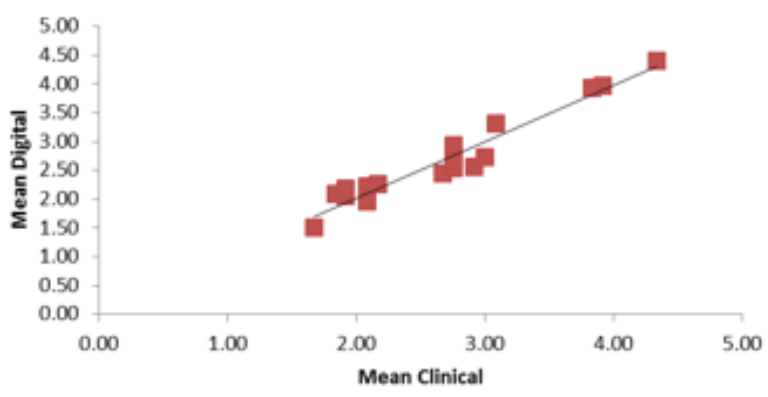

Fig. 6. The correlation between clinical and 3D digital measurement
However, correlation, focusing on the association of changes in two outcomes (often measuring quite different constructs) is distinct from agreement, known as reproducibility (i.e., reliability) between two measurements [8]. Bland and Altman plot was used for agreement evaluation between the two methods (Fig.7).

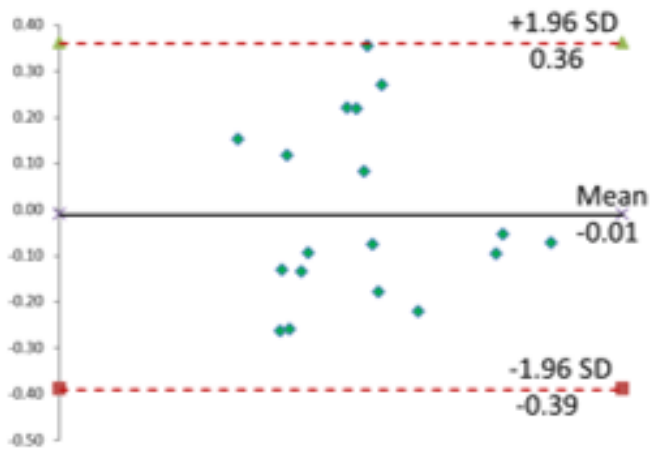

Fig. 7. Agreement between clinical and 3D digital measurements

Also, QQ plot (Quantile-Quantile plot) was performed to evaluate if the variables are normally distributed (Fig. 8).

Correlation and agreement between clinical and digital $2 \mathrm{D}$, and digital 3D versus digital 2D, are presented in Fig.9 and 10.

Periodontal health is a clinical condition related to the absence of progressive attachment loss around the tooth. The classifications of periodontal diseases published by the Consensus of the 2017 World Workshop on the Classification of Periodontal and Peri ImplantDiseases and Conditions are based on measurements of attachment level, probing depth, bone loss and/or degree of inflammation [12]. In the presentstudy, the measurements of the attachment level or the apico-coronal migration of the free gingival margin, parameter needed to be evaluated and register on periodontal chart, was performed using three different methods. 


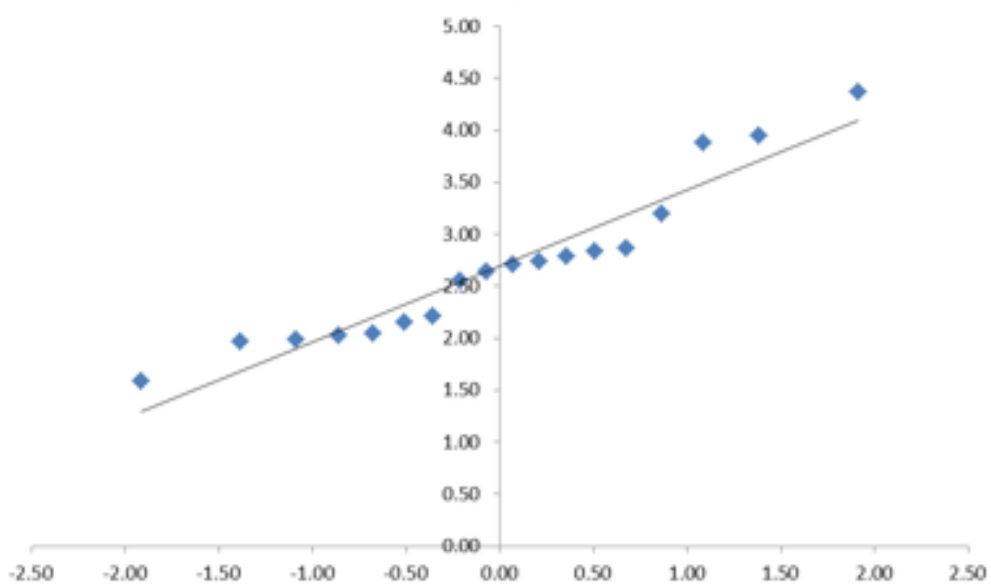

Fig. 8. QQ plot test shows the normal distribution of the variables obtained from clinical and 3D digital measurements (on virtual digital models obtained via intraoral scanning)
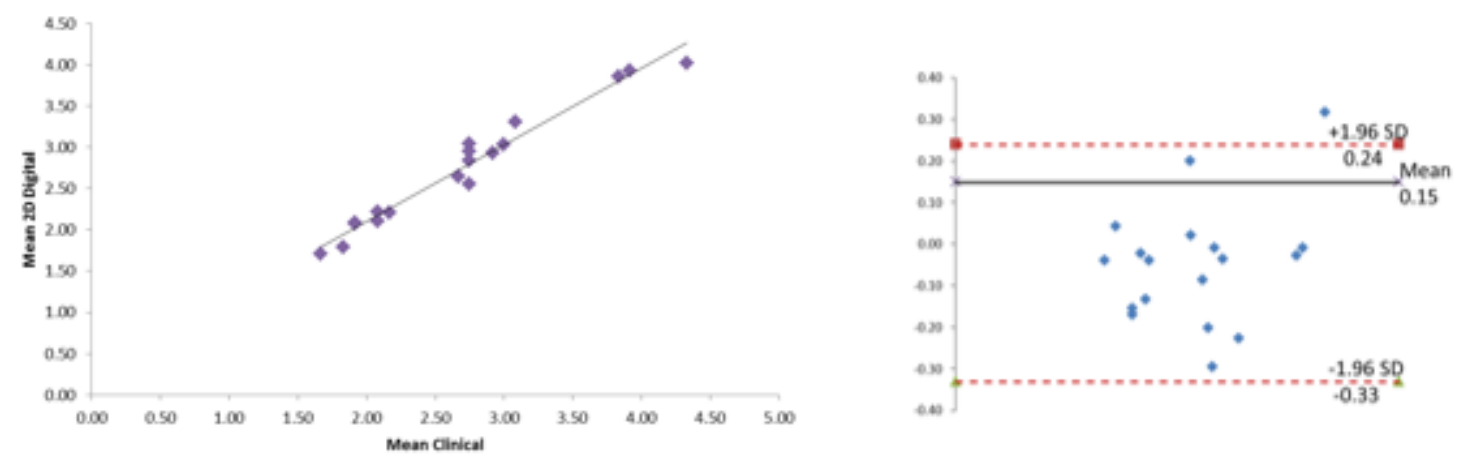

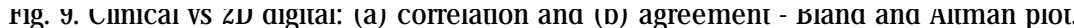
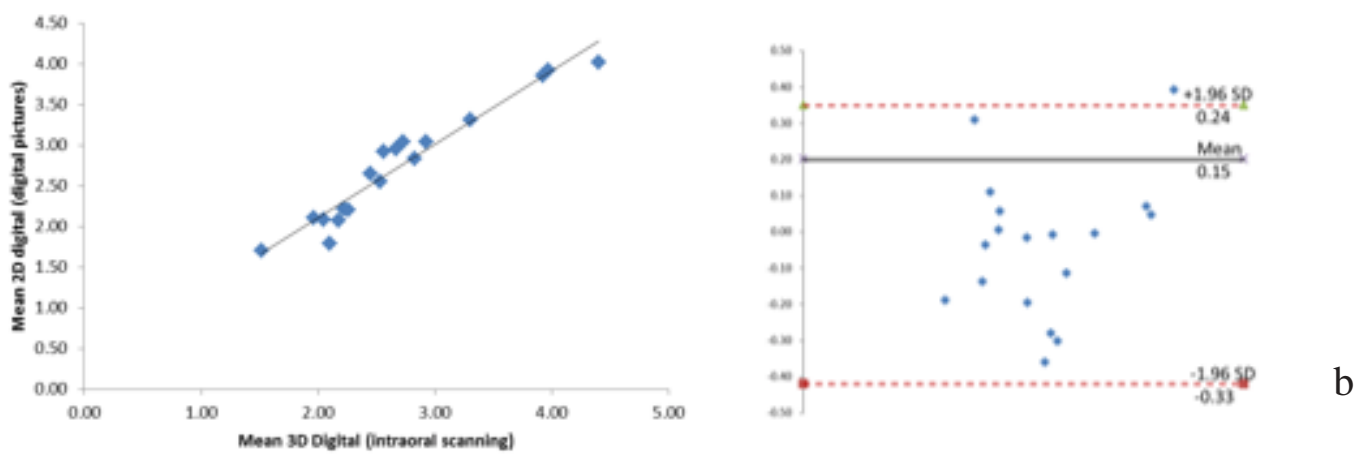

Fig. 10. 3D digital vs 2D digital: (a) correlation and (b) agreement - Bland and Altman plot

The accuracy of the digital measurements of gingival margins was evaluated by the two parameters described by the ISO standard 5725-1 [9]: trueness - referring to the existing deviation between the real dimension of the measured object and the measurement result itself - and precision - referring to the repeatability of the measurements $[10,11]$.

For trueness, the variations between $2 \mathrm{D}$ and $3 \mathrm{D}$ digital measurements and clinical measurements, considered gold standard, were compared pair-wise using Bland and Altman Plots (Fig. 7-9). The mean values for each pair: clinical - 3D digital (Fig. 7), clinical -2D digital (Fig. 9b) and 3D - 2D digital (Fig. 10b) were plotted against the difference of the measurements. The difference for each point, the mean difference, and the confidence limits are illustrated on the vertical axis, while the averages of two measurements are displayed along the horizontal axis. Of the three horizontal lines in the graph, the middle line represents the observed difference in mean values, and the dotted red lines on the top and bottom indicate the $95 \%$ confidence limits within which about $95 \%$ of the differences between the measurements of each method should lie [7]. The difference between the three different measurements was less than $0.4 \mathrm{~mm}$, as follows: between clinical and 3D digital measurements were $\leq 0.35 \mathrm{~mm}$, between clinical and 2D digital were $\leq 0.32 \mathrm{~mm}$ and between $2 D$ and $3 D$ were $\leq 0.39 \mathrm{~mm}$. The extent of variation was assessed using the confidence limits around the mean, as follows: $0.75 \mathrm{~mm}$ for comparison between clinical and 3D digital as well as for 2D digital and 3D digital and 0.57 for clinical versus 2D digital. Mean values were 0.01 for clinical/3D digital and 0.15 for clinical/2D digital and 2D digital/3D digital, mean values closer to zero indicating better agreement between the methods. As it could be observed, clinical and 3D digital measurements scored the closest values.

Regarding precision/repeatability of the measurements, Bland and Altman analysis (Fig. $5 \mathrm{a}-\mathrm{c}$ ) revealed that the differences in measurement between examiners were not clinically significant; the maximum difference did not exceed $0.5 \mathrm{~mm}$ for the clinical measurements, $0.15 \mathrm{~mm}$ for 3D digital and $0.27 \mathrm{~mm}$ for $2 \mathrm{D}$ digital. However, these differences have a broad 95\% confidence intervals extending up to $1.74 \mathrm{~mm}$ for clinical measurements, 1.28 for $3 \mathrm{D}$ digital and 0.72 for $2 \mathrm{D}$ digital. The highest difference in measurement obtained by the two investigators was 
during the clinical evaluation and could be explained by the millimeter calibrated probe.

Intraoral scanning was proved to be a reliable method for measuring the gingival level for diagnosis and clinical monitoring, having a good accuracy as compared to the gold standard (clinical measurements).

In order to evaluate the patients and assess treatment outcome, also to document difficult cases and for medicolegal purpose, physical models are currently used.

As mentioned within the Experimental Section, the material used in dentistry for creating a replica/a model of the oral cavity could be obtained using the dental plaster that is the $\beta$-hemihydrate form of the calcium sulphate (Fig.2). The thermal treatment (calcination) applied to the dehydrate variety of calcium sulphate has a direct effect on the microstructure of the calcium sulphate hemihydrate, as the $\beta$-hemihydrate variety supports highly stressed conditions - water is released as hot steam and the gypsum crystals are smashed during calcination following a specific kinetic mechanism [12]. When certain amount of water is added to the hemihydrate form it releases heat and reverts to the dihydrate form [13].

The recycling loop (Fig.2), which takes place via solution route, is modeled by the chemical processes involving the hydration of hemihydrate, which occurs quite quickly. The last chemical reaction in sequence shows the overall hydration reaction as result of the previous two stages.

$$
\begin{aligned}
& \mathrm{CaSO}_{4}-0: 5 \mathrm{H}_{2} \mathrm{O} \rightarrow \mathrm{Ca}^{2+}+\mathrm{SO}_{4}{ }^{2-}+0.5 \mathrm{H}_{2} \mathrm{O} \\
& \mathrm{Ca}^{2+}+\mathrm{SO}_{4}{ }^{2+}+2 \mathrm{H}_{2} \mathrm{O} \rightarrow \mathrm{CaSO}_{4}-2 \mathrm{H}_{2} \mathrm{O} \\
& \mathrm{CaSO}_{4}-0: 5 \mathrm{H}_{2} \mathrm{O}+1.5 \mathrm{H}_{2} \mathrm{O} \rightarrow \mathrm{CaSO}_{4}-2 \mathrm{H}_{2} \mathrm{O}
\end{aligned}
$$

The $\beta$-hemihydrate forms consist in porous irregular particles, dispersed (Fig. 11). It should be remarked the white color of the dental model generated using the $\beta$ hemihydrate calcium sulphate.

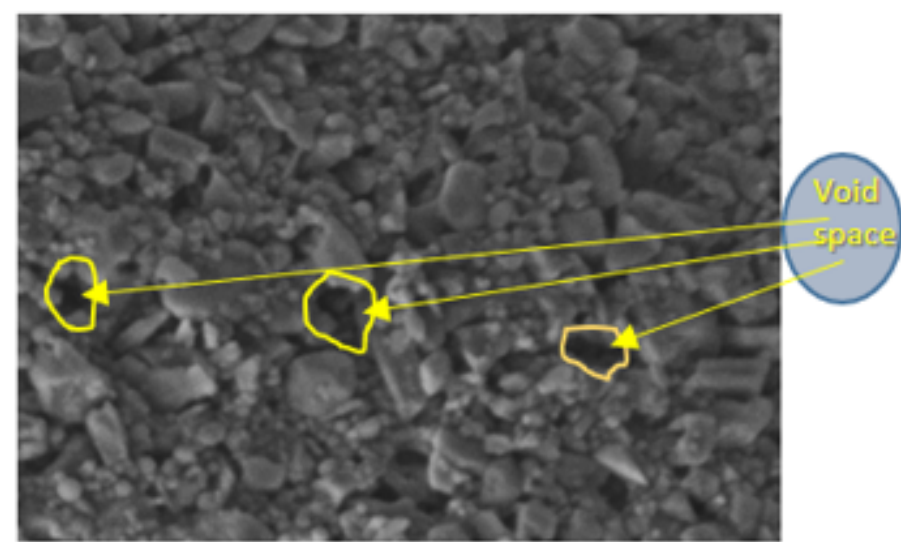

Fig. 11. SEM image of $\beta$-hemihydrate calcium sulphate variety.

The processes occurring during the plaster handling are in direct relation with the hydration reaction (III), resulting gypsum when part of the $b$-hemihydrate and water are consumed as reactants. As consequence, the material volume is given by the chemical shrinkage through the hydration reaction, the un-hydrated $\beta$-hemihydrate gypsum, and the water that remains after the hydration process. However, there is a void space that could contain either water, or air (Fig. 11).

Such void fraction of the material could be associated with a volume increasing due to the reaction shrinkage and the remaining water. The mechanical properties of the plaster are directly influenced by the material's microstructure and as consequence by the hydrating system performance.
The water initiates the setting reaction of the hemihydrate material (Fig.2). The amount of water added assured a creamy mixture that could be afterwards easily manipulated. Although potassium sulphate can be added for accelerating the setting time, it was chosen an alternative solution: creating nuclei of crystallization by adding calcium sulphate dehydrate. The quantity used was $0.5 \%$. Also, sodium chloride has been introduced, which reduced the setting expansion as it provided supplementary sites for crystals growth. The amount of hydration during handling and also the loss of water in time due to evaporation during storage can lead to important volumetric changes in the gypsum models.

Despite of the fact that plaster models are widely used due to requirement of accurate documenting of clinical cases, gypsum is prone to volumetric distortions, physical or chemical damage and requires additional space for storage. Replacing conventional models with virtual models either by direct - digital impression, or indirect -by digitizing the conventional plaster models, have solved the major drawbacks of the gypsum, allowing an accurate record - keeping.

Regarding patient's choice, in our study, the recorded VAS for the 10 patients was 9.5 , illustrating the preference for digital technology when compared to conventional clinical measurements.

Our study is in agreement with other papers suggesting lower variances in gingival recession measurements with the use of digital technology [2]. Intraoral scanning was also proved to be more accurate comparing to the use of plaster models [14], the disadvantages of study casts being solved by the digital ones. The virtual models obtained via intraoral scanning could be used for tracking subtle changes in soft tissue length and volume between dental appointments [15] using a superimposition protocol [16].

Moreover, due to a great accuracy and digital storage (with no volumetric changes whatsoever), it has been suggested that high definition color 3D intra-oral scans could become eventually the new gold standard in periodontal practice for treatmentmonitoring, trend already seen in orthodontics [17].

\section{Conclusions}

Despite of the limitations regarding the reduced number of patients enrolled, the results of our study support the use of 3D optical method for successfully measure the gingival margins with a high degree of reproducibility and reliability and could replace clinical evaluation. A significant advantage that should take into consideration is treatment monitoring that could be performed by superimposing digital models obtained over time.

Acknowledgments: This work was supported by a grant of the Romanian National Authority for Scientific Research and Innovation, CCCDI -UEFISCDI, project number 39/2018 COFUND-MANUNET IIIHAMELDENT, within PNCDI III.

\section{References}

1.DEFERM, J. T., SCHREURS, R., BAAN, F., BRUGGINK, R., MERKX, M. A. W., XI, T., BERGE, S. J., MAAL, T. J. J., Clin Oral Investig, 22, no.3, 2018, p.1303.

2.FAGEEH, H. N., MESHNI, A. A., JAMAL, H. A., PREETHANATH, R. S., HELBOUB, E., BMC Oral Health, 19, art.no.154, 2019.

3.SCHNEIDER, D., ENDER, A., TRUNINGER, T., LEUTERT, C., SAHRMANN, P., ROOS, M., SCHMIDLIN, P., J Esthet Restor Dent, 26, no.3, 2014, p.191.

4.LEHMANN, K. M., KASAI , A., ROSS, A., KAMMERER, P. W., WAGNER, W., SCHELLER, H., J Periodontol; 83, no.1, 2012, p.50. 
5.AYDINYURT, H. S., ERTUGRUL, A. S., Med Sci Discov, 4, no.10, 2017, p.72.

6.EKE, P. I., PAGE, R. C., WEI, L., THORNTON-EVANS, G., GENCO, R. J.,. J Periodontol, 83, no.12, 2012, p.1449.

7.BLAND, J. M., ALTMAN, D. G., Ultrasound Obstet Gynecol, 22, no.1, 2003, p.85

8.LIU, J., TANG, W., CHEN, G., LU, Y., FENG, C., TU, X. M., Shanghai Arch Psychiatry, 28, no.2, 2016, p.115.

9.*** ISO standard 5725-1:1994, ISO, last reviewed in 2018, https:// www.iso.org/standard/11833.html

10.BURDE, A. V., DUDEA, D., CUC, S., MOLDOVAN, M., CAMPIAN, R. S., Mat. Plast., 53, no. 1, 2016, p.65.

11.BURDE, A. V., MANOLE, M., CAMPIAN, R., S., SINESCU, C., BACIU, S., Rev. Chim.(Bucharest), 70, no.7, 2019, p.2344.
12.EFTIMIE, E., SEGAL, E., Thermochim Acta, 111, 1986, p.359.

13.EREMIN, A., PUSTOVGAR, A., PASHKEVICH, S., IVANOVA, I., GOLOTINA, A., Procedia Eng., 165, 2016, p.1343.

14.CHALMERS, E. V., MCINTYRE, G. T., WANG, W., GILLGRASS, T., MARTIN, C. B., MOSSEY, P. A., Cleft Palate-Craniofacial J., 53, no.5, 2016, p.568.

15.LEHMANN, K. M., KASAJ, A., ROSS, A., WILLERSHAUSEN, I., SCHMIDTMANN, I., STAEDT, H., SCHELLER, H., Int J Comput Dent, 14, no.4, 2011, p.297.

16.CRISTACHE, C. M., GURBANESCU, S., Int J Dent, 2017, 4292081.

17.ROSSINI, G., PARRINI, S., CASTROFLORIO, T., DEREGIBUS, A., DEBERNARDI, C. L., Am J Orthod Dentofac Orthop, 149, no.2, 2016, p.161.

Manuscript received: 3.09 .2019 